\title{
炔烃的多组分环化反应选择性合成非芳香五元含硫杂环
}

\author{
胡蓉秦安军* \\ (华南理工大学发光材料与器件国家重点实验室 广东省分子聚集发光重点实验室 \\ 华南理工大学-香港科技大学联合研究院 广州 510640)
}

\section{Selective Synthesis of Non-Aromatic Five-Membered Sulfur Heterocycles via Multicomponent Cyclization of Alkynes}

\author{
Hu, Rong Qin, Anjun*
}

(State Key Laboratory of Luminescent Materials and Devices, Guangdong Provincial Key Laboratory of Luminescence from Molecular Aggregates, South China University of Technology-The Hong Kong University of Science \& Technology, Joint Research Institute, South China University of Technology, Guangzhou 510640)

杂环, 特别是五元含硫杂环, 在天然产物、医药分 子和材料化学等领域广泛存在. 例如艾沙托立宾, 一种 新型的鸟苷类似物, 可作为 TLR7 激动剂 ${ }^{[1]}$; 法罗培南 钠是一种可有效杀死结核分枝杆菌的口服可吸收培南 类抗生素 ${ }^{[2]}$; 苯并草硫醇衍生物 BOT-64 也是一种 $\mathrm{NF}-\mathrm{kB}$ 抑制剂 ${ }^{[3]}$. 在各种制备原料中, 炔烃作为一种重 要的石油化工原料, 近年来伴随着金属催化和有机催化 的蓬勃发展, 已被成功应用于各种不饱和功能杂环分子 的合成. 然而, 由于芳香驱动力的存在, 目前这些反应 大多受限于利用 3 中心 4 电子的 $\pi$ 电子给体[例如 $N=$ $\mathrm{N}-\mathrm{N}, \mathrm{N}=\mathrm{C}(\mathrm{R})-\mathrm{N}, \mathrm{O}-\mathrm{C}(\mathrm{R})=\mathrm{N}$ 和 $\mathrm{S}-\mathrm{C}(\mathrm{R})=\mathrm{N}$ 等 $]$ 与 炔烃反应, 且一般只能构建五元杂环芳香化合物, 如三 唑、咪唑、噁唑和噻唑等(Scheme 1). 因此, 如何构建类 型丰富、结构多样的不饱和杂环化合物仍是该领域的一 个重大挑战.

国内外有机化学家进行了这方面的有益尝试. 2014
年, Gulías 等 $^{[4]}$ 发展了一种铑催化的 [5+2]环加成反应, 直接利用邻乙烯基苯酚和内炔合成七元苯氧一杂环苯并 西平. Hajra 等 ${ }^{[5]}$ 通过铜催化的 2-氨基苯并噻唑与末端炔 烃的氧化偶联反应, 实现了六元苯并噻嗪的合成. 2019 年, Matsubara 等 ${ }^{[6]}$ 报道了镍催化的苯并噻吩与炔烃的 [5+2]环加成反应, 构建了七元环骨架的非芳香苯并硫 杂呋庚因类衍生物. 尽管这一领域已取得一些重要的进 展，但如何利用简单炔烃的多组分反应构建非芳香五元 杂环一直未能突破, 这主要是由于相应的环烯中间体活 化能垒较高, 导致其在热力学上不太稳定.

近日, 华南理工大学化学与化工学院江焕峰和伍婉 卿团队 ${ }^{[7]}$ 在前期关于炔烃化学的研究基础上，利用异硫 氧酸酯、水与简单炔烃的三组分反应，引入了新的 3 中 心 4 电子 $\pi$ 电子体系 [即 $\mathrm{S}-\mathrm{C}(=\mathrm{N})-\mathrm{O}$ 或 $\mathrm{S}-\mathrm{C}(=\mathrm{O})-$ $\mathrm{N}(\mathrm{R})$ 等]，与炔基相结合，成功建立了一种构建非芳香 五元含硫杂环化合物的有效策略 ${ }^{[8]}$. 在质子酸 $/ N$-氯代

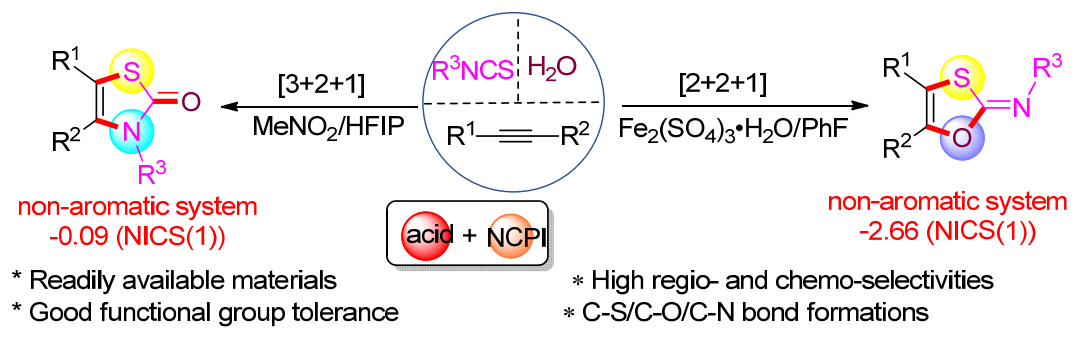

图式 1 利用炔烃、异硫氰酸酯和水的三组分反应选择性合成非芳香五元含硫杂环化合物

Scheme 1 Selective construction of non-aromatic five-membered sulfur-containing heterocycles from multicomponent reaction of alkynes, isothiocyanates and water

* Corresponding author. E-mail: msqinaj@scut.edu.cn. Published online December 30, 2020. 
酞酰亚胺(NCPI)作用下, 异硫㲵酸酯可被水活化, 并被 赋予新的反应活性. 通过 “溶剂开关” 调控中间体 $\mathrm{S}=$ $\mathrm{C}(=\mathrm{N})=\mathrm{O}$ 的生成, 从而选择性得到了 1,3-氧硫醇-2-亚 胺和噻唑-2(3H)-酮衍生物两类非芳香五元含硫杂环.

该反应条件温和, 底物适用性广, 多种取代的芳基 内炔和芳基端炔都能兼容, 同时具有良好的化学选择性 和区域选择性. 机理研究表明该反应经历了水和异硫氰 酸酯的结合、自由基的生成、碳正离子的转化、分子内 环化和脱质子等转化过程.

为了进一步论证该方法的实用性, 该团队通过钯催 化的分子内芳基化反应对所获得的产物进行后续修饰, 成功构建了 $[6,6,6,5]$ 多并环结构衍生物(Scheme 2).
最后，该团队进一步探索了 1,3-氧硫醇-2-亚胺类衍 生物的功能. 以具有发光性能的化合物 7 为例, 将其与 12 种常用的金属离子, 包括 $\mathrm{Ag}^{+} 、 \mathrm{Co}^{2+} 、 \mathrm{Cu}^{2+} 、 \mathrm{Fe}^{2+} 、$ $\mathrm{Fe}^{3+} 、 \mathrm{~K}^{+} 、 \mathrm{Li}^{+} 、 \mathrm{Mg}^{2+} 、 \mathrm{Mn}^{2+} 、 \mathrm{Na}^{+} 、 \mathrm{Zn}^{2+}$ 和 $\mathrm{Pd}^{2+}$ (阴离 子均为 $\mathrm{OAc}^{-}$)等进行作用, 结构显示只有金属 $\mathrm{Pd}^{2+}$ 离子 导致其苂光猝灭，而其它的金属离子对其苂光发射强度 没有产生明显影响, 有些甚至使得荧光发射增强(图 1). 这表明 1,3-氧硫醇-2-亚胺类衍生物在金属 $\mathrm{Pd}^{2+}$ 离子检 测方面具有潜在的应用前景. 值得指出的是，该反应直 接将水作为反应物之一来构筑功能杂环，相信通过进一 步优化也可发展绿色单体(水)参与的炔类单体的高效聚 合反应 ${ }^{[9-10]}$.

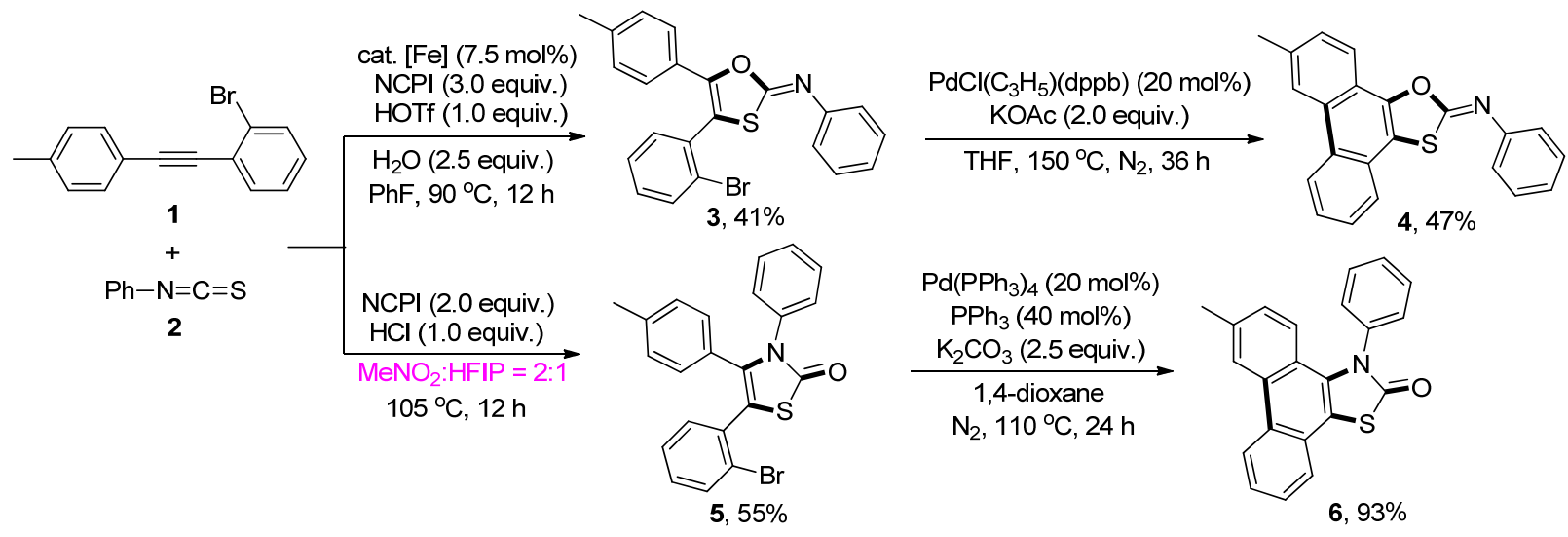

图式 2 多并环化合物的构建

Scheme 2 Construction of polycyclic heterocycles
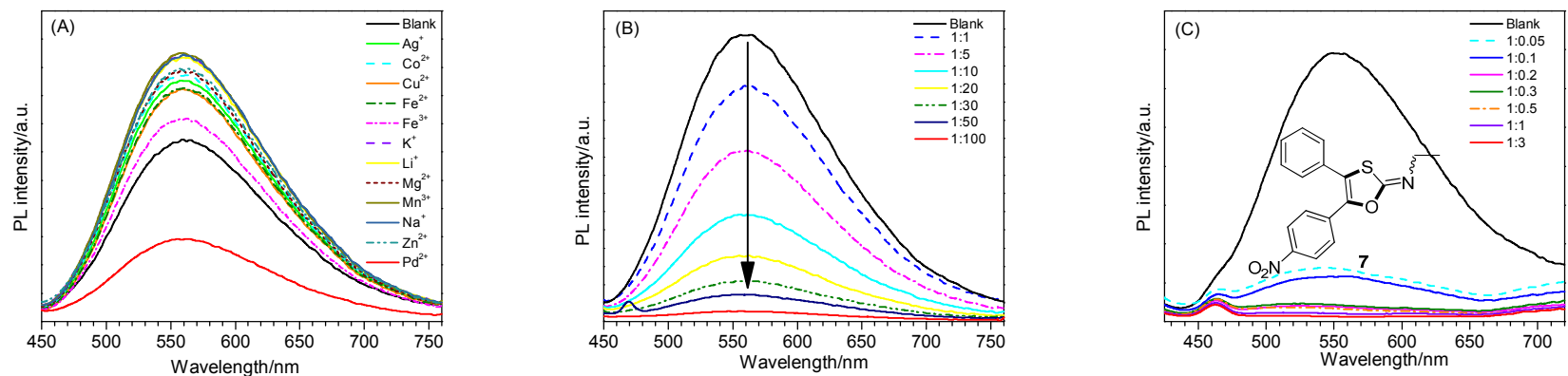

图 1 化合物 7 的苂光发射光谱随着不同离子种类 $(\mathrm{A})$ 和 $\mathrm{Pd}^{2+}$ 浓度 $(\mathrm{B}, \mathrm{C})$ 的变化曲线

Figure 1 Changes in the photoluminescence (PL) spectra of 7 with different ions (A) and $\mathrm{Pd}^{2+}$ concentration $(\mathrm{B}, \mathrm{C})$

\section{References}

[1] Horsmans, Y.; Berg, T.; Desager, J.-P.; Mueller, T.; Schott, E.; Fletcher, S. P.; Steffy, K. R.; Bauman, L. A.; Kerr, B. M.; Averett, D. R. Hepatology 2005, 42, 724.

[2] Dhar, N.; Dubée, V.; Ballell, L.; Cuinet, G.; Hugonnet, J. E.; Signorino-Gelo, F.; Barros, D.; Arthur, M.; McKinney, J. D. Antimicrob. Agents Chemother. 2015, 59, 1308.

[3] Venkateswararao, E.; Le Tuan Anh, H.; Sharma, V. K.; Lee, K.-C.; Sharma, N.; Kim, Y.; Jung, S.-H. Bioorg. Med. Chem. Lett. 2012, $22,4523$.

[4] Seoane, A.; Casanova, N.; Quinones, N.; Mascareñas, J. L.; Gulías,
M. J. Am. Chem. Soc. 2014, 136, 834.

[5] Mitra, S.; Chakraborty, A.; Mishra, S.; Majee, A.; Hajra, A. Org. Lett. 2014, 16, 5652

[6] Inami, T.; Takahashi, T.; Kurahashi, T.; Matsubara, S. J. Am. Chem. Soc. 2019, $141,12541$.

[7] Tang, X.; Wu, W.; Zeng, W.; Jiang, H. Acc. Chem. Res. 2018, 51, 1092.

[8] Yu, W.; Zhu, B.; Shi, F.; Zhou, P.; Wu, W.; Jiang, H. Angew. Chem. Int. Ed. 2021, 60, 13131322.

[9] Zhang, J.; Wang, W. J.; Liu, Y.; Sun, J. Z.; Qin, A. J.; Tang, B. Z. Macromolecules 2017, 50, 8554

[10] Liu, Y.; Qin, A. J.; Tang, B. Z. Prog. Polym. Sci. 2018, 78, 92.

(Lu, Y.) 